\section{Prick your mind with Allergy!}

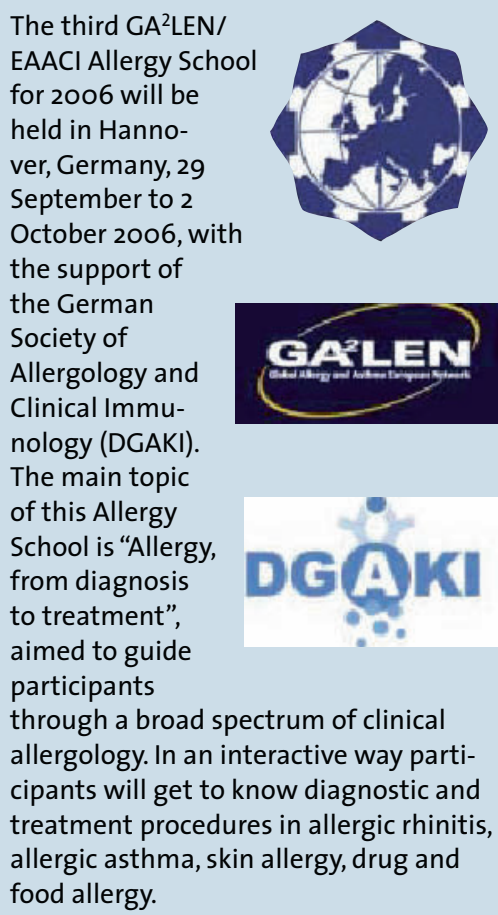

For the first time voting procedures are implemented within this Allergy School to guarantee a lively and interactive participation. In addition, interactive workouts on clinical case reports are offered.

Submission of abstracts for poster presentation is not obligatory but strongly encouraged. Abstract quality and poster presentation will be taken into account for awarding 40 poster prizes of $100 €$. A limited number of travel grants will be provided for EAACI Junior members (under 35 of age) and GA2 LEN PhD students with an abstract (abstract quality will be taken into account).

For Junior members an all-inclusive fee of $150 €$ is required for registration. All meals, social events and shared accommodation (3 nights) in a double room are included. Non-juniors pay an all-inclusive fee of $600 €$. All meals, social events and accommodation (3 nights) in a single room are included.

On-line registration is open until 25 September, 2006, but please register as soon as possible since the number of places to this course is limited to go persons. No registrations will be accepted after this date!

For abstract submission and registration please visit the EAACI website.

www.eaaci.net

\title{
Münchner Wissenschaftlerin ausgezeichnet
}

$\mathrm{P}$ riv.-Doz. Dr. Claudia Traidl-Hoffmann, Leiterin der Arbeitsgruppe „Zelluläre Immunologie“ am Zentrum Allergie und Umwelt der Klinik und Poliklinik für Dermatologie und Allergologie am Biederstein der Technischen Universität München ist die diesjährige Preisträgerin des mit 10.000 Euro dotierten Allergopharma Award. Prof. Dr. Ulrich Wahn, EAACI Past President, und Joachim Ganzer, Geschäftsführer von Allergopharma, überreichten den Preis im Rahmen des diesjährigen Kongresses der EAACI in Wien.

Traidl-Hoffmann wurde für ihre Untersuchungen zu Pollen-assoziierten Lipidmediatoren (PALMs) geehrt. In einem multidisziplinären Team hatte sie herausgefunden, dass Phytoprostan $\mathrm{E}_{1}$ - ein Linolensäurederivat, das in wässrigen Pollenextrakten von der Arbeitsgruppe identifiziert wurde - die Produktion von Interleukin-12 in dendritischen Zellen hemmt. Dieses Zytokin spielt eine wichtige Rolle bei der Induktion einer „normalen“ TH1-Antwort. Dies weist darauf hin, dass Pollen neben den eigentlichen Allergenen noch weitere Werkzeuge besitzen, die eine allergische Reaktion beeinflussen können. Bemerkenswert ist die Tatsache, dass Bäume aus Gebieten mit starker Umweltbe-

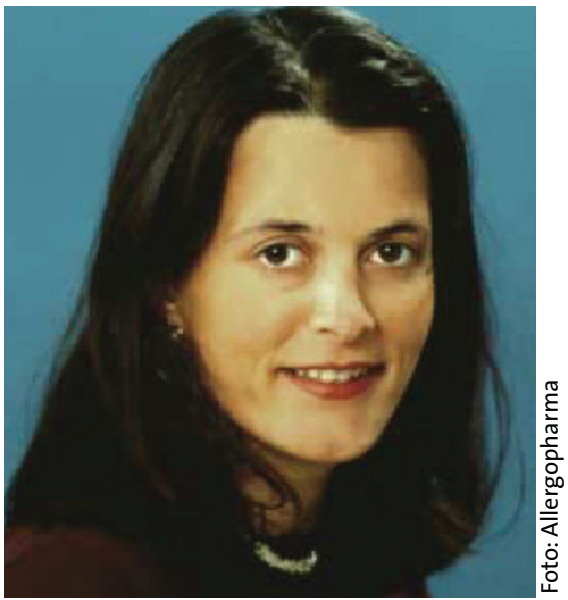

Priv.-Doz. Dr. Claudia Traidl-Hoffmann ist die Preisträgerin des Allergopharma Award 2006.

lastung deutlich größere Mengen dieser PALMs freisetzten. Damit könnte die höhere Prävalenz von Allergien in Gebieten mit starker Luftverschmutzung erklärt werden.

Der Allergopharma Award wurde erstmalig 2001 in Zusammenarbeit mit der EAACI vergeben. Mit dem Preis werden die Forschungsarbeiten junger Akademiemitglieder ausgezeichnet, die auf dem Gebiet der allergischen Entzündungen sowie der spezifischen Immuntherapie tätig sind.

$G C I$

\section{Neues Graduiertenkolleg in Hannover}

$\mathrm{D}$ ie Deutsche Forschungsgemeinschaft (DFG) fördert das neue Graduiertenkolleg „Regulation der allergischen Antwort in Lunge und Haut" der Medizinischen Hochschule Hannover $(\mathrm{MHH})$ für die kommenden viereinhalb Jahre mit knapp einer Million Euro. Mit den Fördermitteln werden zwölf Stellen für Doktoranden, eng vernetzt mit dem internationalen MD-/ PhD-Programm der $\mathrm{MHH}$, eingerichtet. In dem Graduiertenkolleg arbeiten die $\mathrm{MHH}$-Abteilungen Dermatologie und Venerologie, Pneumologie, Kinderheilkunde, pädiatrische Pneumologie und Neonatologie, funktionelle und angewandte Anatomie sowie Gastroenterologie, Hepatologie und Endokrinologie zusammen. Hinzu kommt noch das Fraunhofer Institut für Toxikologie und experimentelle Medizin (ITEM) in Hannover.

„Die an dem Graduiertenkolleg beteiligten Forschergruppen spiegeln das interdisziplinäre Wesen der Allergie wider, die sich mit all ihren Auswirkungen und Ursachen in vielen Fachbereichen findet", betont der Sprecher des neuen Kollegs, Prof. Dr. Thomas Werfel, leitender Oberarzt der Klinik für Dermatologie und Venerologie der MHH. 